\title{
Motion Simulation of Hydraulic Support Based on Unity 3D
}

\author{
Ale $\mathrm{Li}^{\mathrm{a}}$, Xiaowen Zheng ${ }^{\mathrm{b}}$, Wei Wang ${ }^{\mathrm{c}}$ \\ China University of Mining \& Technology(Beijing), Beijing, China \\ akdliale@163.com, 'bxw@cumtb.edu.cn, cwangw2016@126.com
}

Keywords: virtual reality, hydraulic support, Unity 3D, motion simulation

\begin{abstract}
With the rapid development and extensive application of virtual reality technology, many enterprises use this technology to enhance the competitiveness of their products. Using three-dimensional modeling software Pro/E, 3ds Max, virtual reality software Unity 3D and object-oriented programming technology, the three-dimensional modeling and motion simulation of hydraulic support can be realized, and the virtual hydraulic support can be controlled by human-computer interaction.
\end{abstract}

\section{Introduction}

As a new subject of the information sciences, virtual reality technology is widely used in the field of military, manufacturing, entertainment, medical, information system with its unique advantage. The three-dimensional virtual simulation system is powerful, which can not only display products visually via the Internet, but also achieve real-time control effects through human-computer interaction, this plays an important role for users to understand the structure and function of products, the virtual reality software Unity 3D is a good platform. This paper is the research on motion simulation system of hydraulic support based on Unity 3D.

\section{A Brief Introduction of Unity 3D}

As a cross-platform engine of game developing, Unity 3D has the intuitive environment for editing games. Its biggest advantage is being cost-effective, and it can be released in the way of web browsing, which means users can enjoy them without downloading the client. The virtual simulation system of hydraulic support designed through Unity 3D has the interactive features which can't be acquired through other three-dimensional modeling software, so it perfectly meets the requirements of interactive access and realistic performance in the three-dimensional virtual presentation of industrial products.

\section{Research Status at Home and Abroad}

Three-dimensional display and motion simulation through virtual reality technique has got preliminary application overseas, today the basic research in this field is mainly concentrated in these four aspects: perception, user interface, background software and hardware. In recent years, in the studios about virtual reality technology of motor companies such as American General and Ford in the US, people can see that there are various kinds of innovative equipment and tools, the engineers are doing experimental works with tools like helmet and inductive gloves to generate three-dimensional prototype image of the car on the workbench.

Compared with developed countries in the study of this subject, we still have a long way to go because it started too late for us, but there are also quite a few people have made great achievements. For example, ZhuHuijuan from NJUST adopted modeling tools including Unity 3D and 3ds Max, she was successful to reach interaction through programming languages JavaScript and C\#. Finally she finished to design the virtual campus roaming system by the integration with Html ${ }^{[1]}$.

\section{Development Process}


For the specific development process of motion simulation system of hydraulic support mentioned in this paper, firstly, create a virtual model of the hydraulic support which is equivalent to the physical one, and then calculate motion parameters according to the movement of related component and express them by script. So we can achieve human-computer interaction when the script is attached to the hydraulic support model, and finally achieve the purpose of dynamic presentation. After all of these are done, we can have the performance testing, and finally we get the executable files and network files. The brief development process is shown in Fig.1.

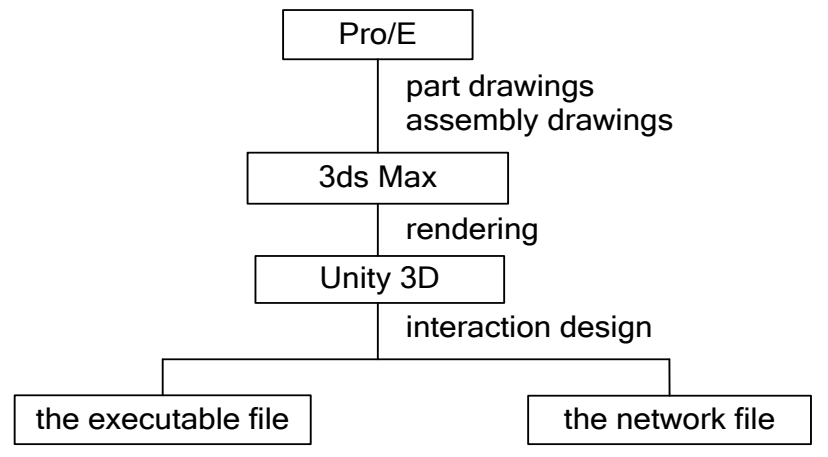

Fig.1. development process of simulation system of virtual hydraulic support

3.1 three-dimensional modeling of hydraulic support

The object of study is the type of four columns hydraulic support ZZ4400/17/35. A hydraulic support is mainly composed by the base, linkages, column, shield beam, roof beam etc. According to the specific structure parameters of hydraulic support, we can build the three-dimensional (hereinafter short for 3D) models by Pro/E from the bottom up. This means that we should draw every single part of hydraulic support in 3D model, and then assembly them all according to their constraint relationship ${ }^{[2]}$.

\section{2 rending and import of model}

The first thing is to import the model of hydraulic support to 3ds Max, then do some renderings and color configuration according to different materials of the hydraulic support model. Because the model in this paper is relatively simple, there is few strict restriction for materials during the later motion simulation, and we don't need to do any change when faced with the work of selecting materials, so let it be the type of Standard. After that, import the file into Unity 3D with the form . $f b x$ which could be recognized by the software, in the end, add necessary lighting and camera. 3.3 motion processing of components

To realize various kinds of movements of the virtual hydraulic support, we just need to deal with the parent-child relationship of related components, then calculate the position coordinates and express them by script in Unity 3D. The parent object has child objects, child objects can also contain additional child objects, there is an inheritance relationship between them. When the father object moves, child objects will follow the same movement, while when the child object moves, the father object will remain its original gesture. This provides great convenience for programming with JavaScript in the next step ${ }^{[3]}$.

So, after handling the parent-child relationship and coordinate of all components, select the back link as a driving component, the movement of other parts of hydraulic support can be achieved when we just need to attach the script to back link. The instantaneous position coordinates and the angle of each moving part can also be displayed on the interface through text. According to the relationship among various parts of hydraulic support and convenient calculation requirements, a part of parent-child hierarchical relationship is set as shown in Fig.2. 


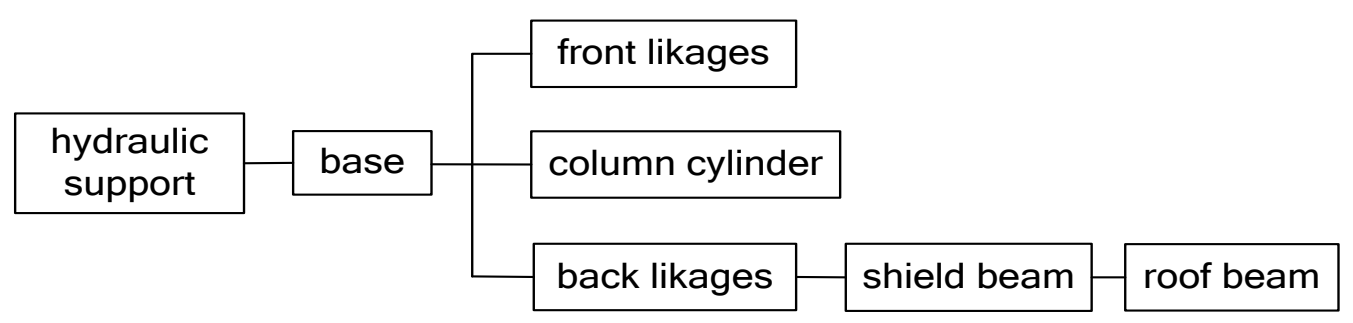

Fig.2. parent-child relationship

When doing the motion simulation of hydraulic support, its movements include the rotation of linkages, the glide between columns, the rotation of shield beam and roof beam, even the movement of the whole hydraulic support. These movements are in a plane, it's better to select the right side view plane of hydraulic support to analysis. About the calculation of coordinates of components, there is simple introduction as shown in Fig.3.

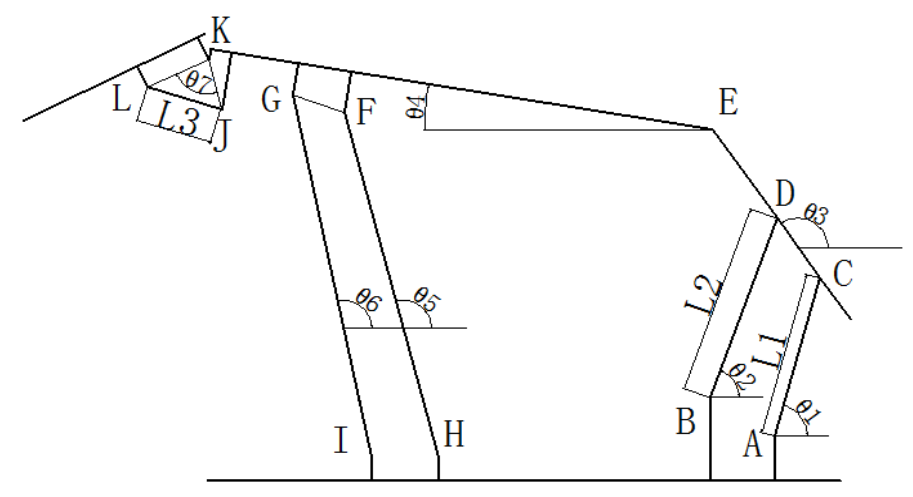

(a)

Fig.3. motion diagram of hydraulic support

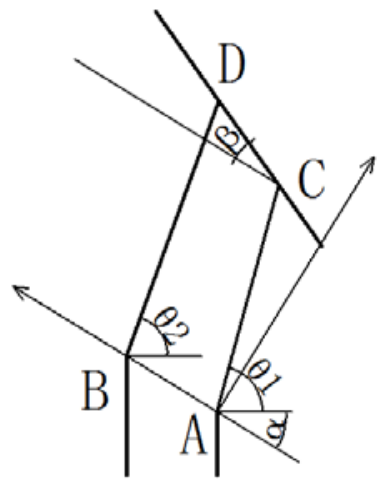

(b)

As shown in Fig.3(a), this is a motion diagram of hydraulic support. First of all, select point A in the axis of rotation of hydraulic support's back link as the origin of hydraulic support in a virtual environment, the relevant calculations begin at this place and gradually go up. As shown in Fig.3(b), the part constitute of point $\mathrm{A}, \mathrm{B}, \mathrm{C}, \mathrm{D}$ is a four-bar link mechanism. When we know the coordinate of point $B$, the length of $C D$, the length of link $A C$ and $B D$, and the angle $\theta_{1}$ of link AC, that we can figure out the angle $\theta_{2}$ of link $B D$, which can further figure out the coordinate of point $C$ and point $\mathrm{D}$. The angle $\theta_{3}$ of shield beam is easy to get according to the coordinate of point $C$ and point $\mathrm{D}$. Combined with the parent-child relationship, for the same reason, we will get all the parameters showed in Fig.3. At last, write these position parameters in the script, the whole virtual movement of each component can be reached through the rotation of back link, thus, it achieves the purpose of motion simulation.

\section{4 interaction design}

Virtual hydraulic support is a 3D simulation object generated by computer, users can take a series of operations in the virtual object (hydraulic support) through controlling the mouse and keyboard. Interaction is the key to controlling a hydraulic support, while script writing is the way to achieve human-computer interaction, attach the script to corresponding GameObject (the active components of hydraulic support in this paper) can achieve dynamic interaction in 3D scene. Simple interaction can be realized with JavaScript, so this paper is using the JavaScript to program the code of interaction.

To realize a specific function of hydraulic support through a key on the keyboard, for example, to control the whole advancement of hydraulic support by key ' $W$ ', it can be easily achieved through

JavaScript like this:

var speed $=30$;

function Update ()\{

if(Input.GetKey(KeyCode.W)) \{

transform.Translate(Vector3.forward*Time.deltaTime*speed); 
\}

Through the above process, when finish all the script, finally we can release our product into corresponding executable file and network file in Unity 3D. The final display interface is shown in Fig.4.

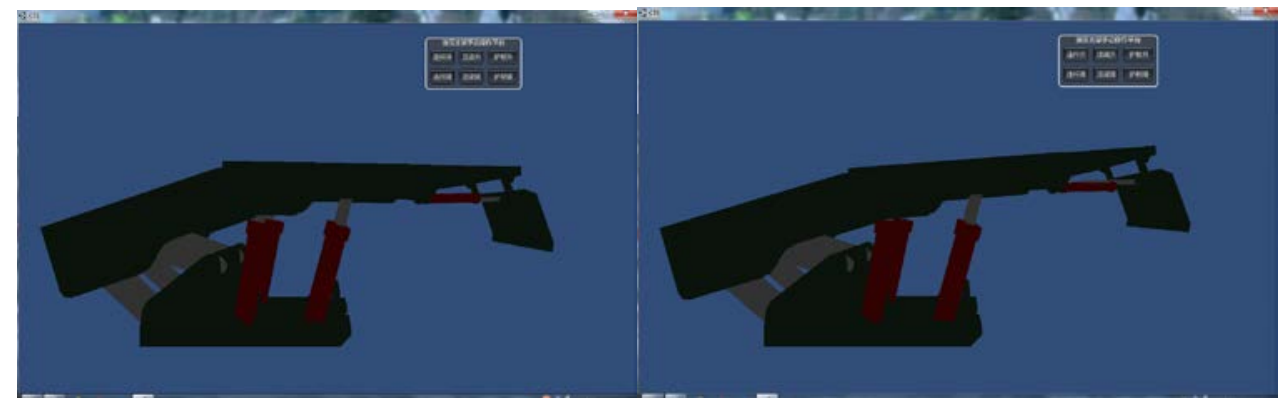

(a)

(b)

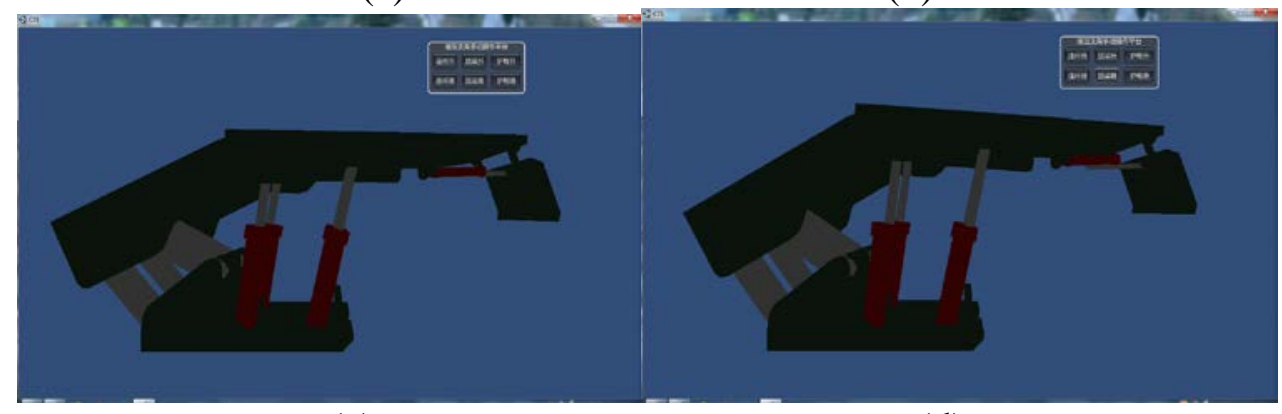

(c)

(d)

Fig.4. display effect

\section{Conclusion}

Using interactive virtual reality software Unity 3D, the simulation of hydraulic support was finished. It has a auxiliary function and practical significance of production practice and the hydraulic support's virtual demonstration, it also has great help to us to understand the working conditions of hydraulic support. Moreover, apply virtual reality technology to a wider field, such as virtual wandering system, it can let us observe the virtual space in the first perspective. And online exhibition of industrial products, it can enhance the competitiveness of products, this is a very important way for customers to understand and become familiar with the product.

\section{Reference}

[1] Zhuhuijuan. Virtual Roaming System Based on Unity 3D[J]. Computer Systems \& Applications, 2012, 21(10): 36-40.

[2] Fankai, Zhangdongjie etc. Three-dimensional Modeling and Analysis of Hydraulic Support Based on Pro/E[J]. Science and Technology Innovation Herald, 2011, 24: 45-46.

[3] Zhangwenlei, Zhengxiaowen etc. Study on hydraulic Support's Working State Based on Virtual Reality[J]. Coal Mine Machinery, 2012, 33(10): 72-74.

[4] Wuyafeng. Game Developing Details \& Typical Cases by Unity 3D[M]. Beijing:Posts \& Telecom Press. 2012.

[5] Qiujiansong. Research on Real-time Virtual Simulation System Based on Unity 3D[J]. Practical Electronics, 2012(12). 